LCLS-TN-05-6

February 2005

\title{
Direct Comparison of Brookhaven Reflectivity Measurements with Free-Electron Theory*
}

\author{
Karl L.F. Bane and Gennady Stupakov \\ Stanford Linear Accelerator Center, \\ Stanford University, Stanford, CA 94309 \\ Jiufeng $\mathrm{Tu}$ \\ Department of Physics, \\ The City College of New York, \\ New York, NY 10031
}

*Work supported by the Department of Energy, contract DE-AC02-76SF00515 


\section{REFLECTIVITY AND FREE-ELECTRON PARAMETERS}

The reflectivity at normal incidence of copper and aluminum samples was recently measured over a large frequency range at Brookhaven by one of us (JT) [1]. Then using the Kramers-Kroning integrals, and assuming the free-electron model of conductivity, the dependence of conductivity on frequency was obtained. The results seemed to suggest, for example, that the dc conductivities of the copper and evaporated aluminum samples are a factor of 3 lower than expected [1]. We propose in this report, instead, directly fitting the free-electron model to the low frequency end of the reflectivity data. This fitting does not depend on the higher frequency results and on Kramers-Kronig integrations, but it does assume that the data at the low frequency end is sufficiently accurate. Note that for our LCLS wakefield studies, it is only over these (relatively) low frequencies that we need to know the electrical properties of the metals.

The equations that relate reflectivity $R$ with the free electron parameters dc conductivity $\sigma$ and relaxation time $\tau$ are:

$$
\begin{gathered}
\tilde{\sigma}=\frac{\sigma}{1-i k c \tau}, \\
\tilde{n}=\sqrt{\tilde{\epsilon}}=\sqrt{1+\frac{4 \pi i \tilde{k} c}{\omega}}, \\
R=\left|\frac{\tilde{n}-1}{\tilde{n}+1}\right|^{2} .
\end{gathered}
$$

The parameters are ac conductivity $\tilde{\sigma}$, index of refraction $\tilde{n}$, dielectric constant $\tilde{\epsilon}$, and wave number $k=\omega / c$, with $\omega$ frequency and $c$ the speed of light.

In Fig. 1 we show the ideal behavior of $R$ for a reasonably good conducting metal, where $\sigma=0.12 \times 10^{17} / \mathrm{s}$ and $\tau=0.55 \times 10^{-14} \mathrm{~s}$ (solid line); these parameters are, respectively, $2 \%$ $(\sigma)$ and $20 \%(\tau)$ of the nominal values for copper. The parameters were chosen so that the important features of $R(k)$ could be seen easily in one plot. We see 3 distinct regions: (1) for low frequencies, $k \lesssim 1 / c \tau, R$ continually decreases, with positive curvature, and with a low frequency asymptote of $(1-\sqrt{2 k c / \pi \sigma}) ;(2)$ for intermediate frequencies the reflectivity is

nearly constant, $R \approx(1-\sqrt{1 / \pi \sigma \tau}) ;(3)$ for $k \gtrsim k_{p}=\sqrt{4 \pi \sigma / c^{2} \tau}$, the plasma frequency of the metal, $R$ quickly drops to zero. The dashed lines in Fig. 1 give the analytic guideposts 
for the 3 regions. Note that it is only in the first and part of the second region that we can expect the free electron model to have validity in real metals; at higher frequencies the effects of absorption bands and other physics will distort the $R(k)$ curve. In principle, knowing $R$ accurately in the entire 1st region suffices for obtaining the free-electron parameters $\sigma$ and $\tau$; in practise, however, knowing it also in the 2 nd region gives us more confidence in the model and especially in the value of $\tau$.

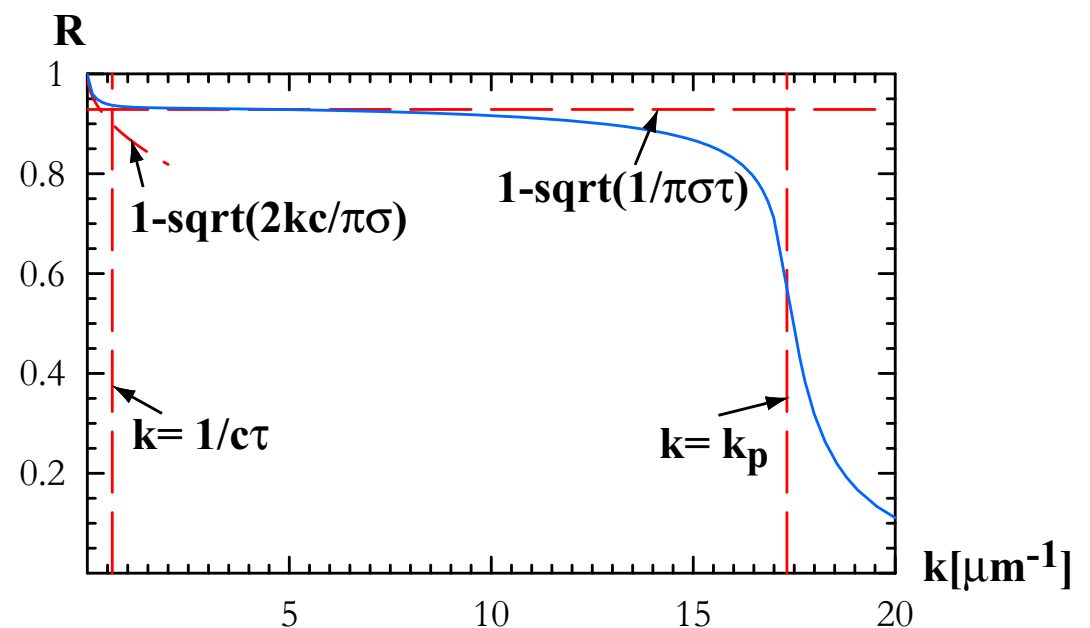

FIG. 1: Frequency $k$ vs. reflectivity $R$ for a metallic conductor, assuming the free electron model (solid line). For this example $\sigma=1.2 \times 10^{16} / \mathrm{s}$ and $\tau=5.4 \times 10^{-15} \mathrm{~s}$. Analytic guideposts are also given (dashes).

\section{ANALYSIS OF DATA}

In Fig. 2 we present the reflectivity of copper and evaporated aluminum, as obtained by the recent Brookhaven measurements. We see in $R$ a possible correct dependence at very low frequencies $((1-R) \sim \sqrt{k})$. But this is followed by a linear decrease, not a constant dependence, which is not consistent with the free-electron model with reasonable parameters. When comparing the $\mathrm{Al}$ curve with a summary plot of $\mathrm{Al}$ reflectivity measurements performed in 1980 by Shiles, et al [2], one can see that the earlier measurements also have a slope in the second region of $R$, but that the slope is a factor of 2 less steep; in addition, there is a pronounced absorption spike near $k=7.5 \mu \mathrm{m}^{-1}$ (beyond our region of interest) that is 
only weakly suggested in the recent measurements. We wonder if these differences are due to sample variability. In the $\mathrm{Cu}$ curve of Fig. 2 we again see a slope in $R$ at low frequencies, followed by a sudden drop at $k=1.5 \mu \mathrm{m}^{-1}$ (beyond our region of interest); these also are not consistent with the free electron model. The onset to interband absorption is seen at $10 \mu \mathrm{m}^{-1}$ (as also seen in Ashcroft and Mermin's Solid State Physics, p. 297 [3]).

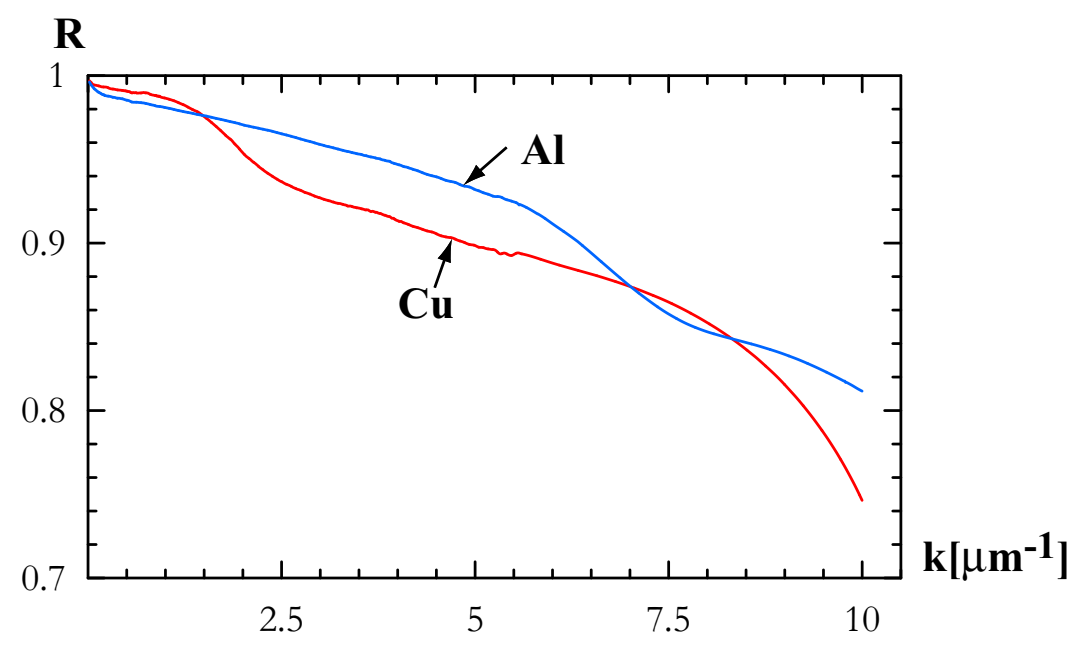

FIG. 2: Measurement results from Brookhaven: frequency $k$ vs. reflectivity $R$ for copper and evaporated aluminum.

These reflectivity measurements are important to the LCLS for verifying resistive wall wakefield calculations in the undulator region [4]. In this region the longitudinal bunch shape is rather rectangular, with an rms length of $20 \mu \mathrm{m}$, but with the addition of narrow "horns" at the head and tail of the distribution. The equivalent wavelength range is $10-100 \mu \mathrm{m}$. We are thus primarily interested in the behavior of $R$ for $k \approx 0.06-0.6 \mu \mathrm{m}^{-1}$, which is the very low frequency end of the data, and the region over which we will try to fit. One thing very positive about the latest measurements, is that, unlike data that we have seen in the literature, the reach is to very low frequencies, and we have hope of fitting to Region 1 type behavior.

The aluminum comparison over nearly twice the region of interest is given in Fig. 3. Blue repeats the measured results. The result of the free electron model, with $\sigma=3.35 \times 10^{17} / \mathrm{s}$ and $\tau=0.75 \times 10^{-14} \mathrm{~s}$ (at $295 \mathrm{~K}$ ) taken from Ashcroft and Mermin [3], is given in green. Note that these parameters agree with a 1983 paper that collected data from many earlier reports, 
and then fitted to the data (though there is no data below $0.2 \mu \mathrm{m}^{-1}$ ) [5]. Not knowing what to do with Region 2, we fit to the free electron model in the first, low frequency region only. The result is $\sigma=(2.12 \pm .02) \times 10^{17} / \mathrm{s}(0.63$ nominal $)$ and $\tau=(0.58 \pm .015) \times 10^{-14} \mathrm{~s}(0.78$ nominal); see the red curve in Fig. 3. We note that the curve fits the data very well up to $0.5 \mu \mathrm{m}^{-1}$, that $\sigma$ is less than ideal by a factor of $2 / 3$ (which is plausible), and that $\tau$ is near nominal. The measurements suggest that the simple free electron model breaks down for $k>0.5 \mu \mathrm{m}^{-1}$.

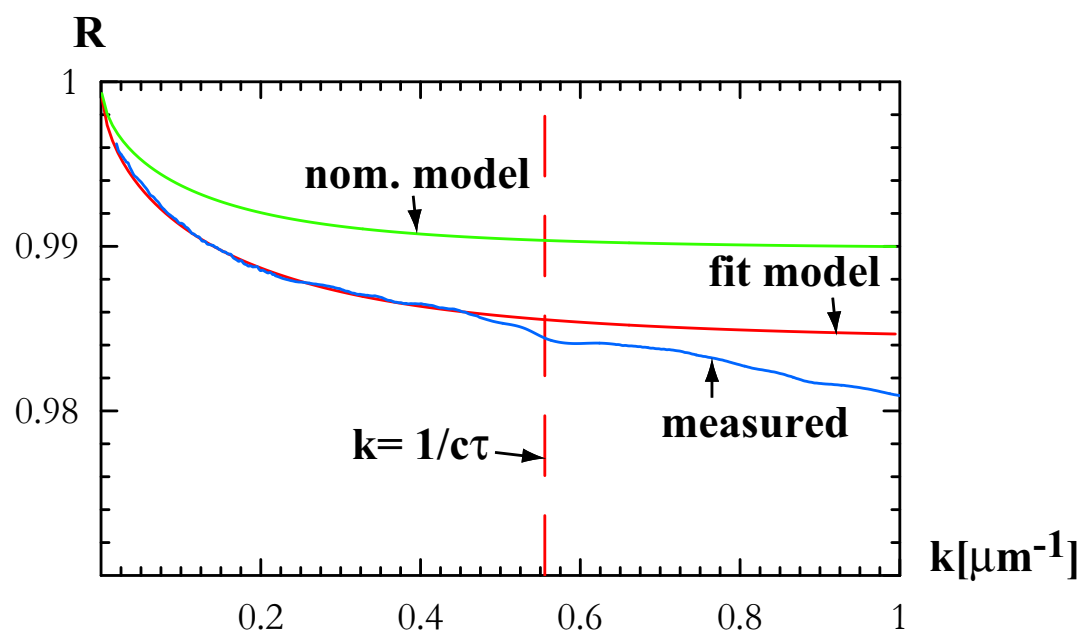

FIG. 3: Aluminum reflectivity: comparison of measurements (blue) with calculations using nominal $\sigma, \tau$ (green); and fitted values: 0.63 nominal $\sigma, 0.78$ nominal $\tau$ (red). The position of $k=1 / c \tau$ for the fit is also shown.

The copper comparison is shown in Fig. 4. Shown are the measured data (blue) and the calculations (green), where we have used parameters $\sigma=5.26 \times 10^{17} / \mathrm{s}$ and $\tau=2.52 \times 10^{-14} \mathrm{~s}$ (at $295 \mathrm{~K}$ ). These parameters were again obtained from Ashcroft and Mermin; they are also in agreement with Lenham and Treherne (though there is no data below $0.3 \mu \mathrm{m}^{-1}$ ) [6]. As before we fit in the low frequency region only. The result is $\sigma=(3.47 \pm .04) \times 10^{17} / \mathrm{s}(0.66$ nominal $)$ and $\tau=(1.68 \pm .025) \times 10^{-14} \mathrm{~s}(0.67$ nominal $)$; see the red curve in Fig. 4 . We again see agreement at the very low frequency end of the plot up to $0.25 \mu \mathrm{m}^{-1}$, though the data is less smooth then before. Note that in this case the agreement breaks down far below the upper end of the desired frequency range $\left(0.6 \mu \mathrm{m}^{-1}\right)$. 


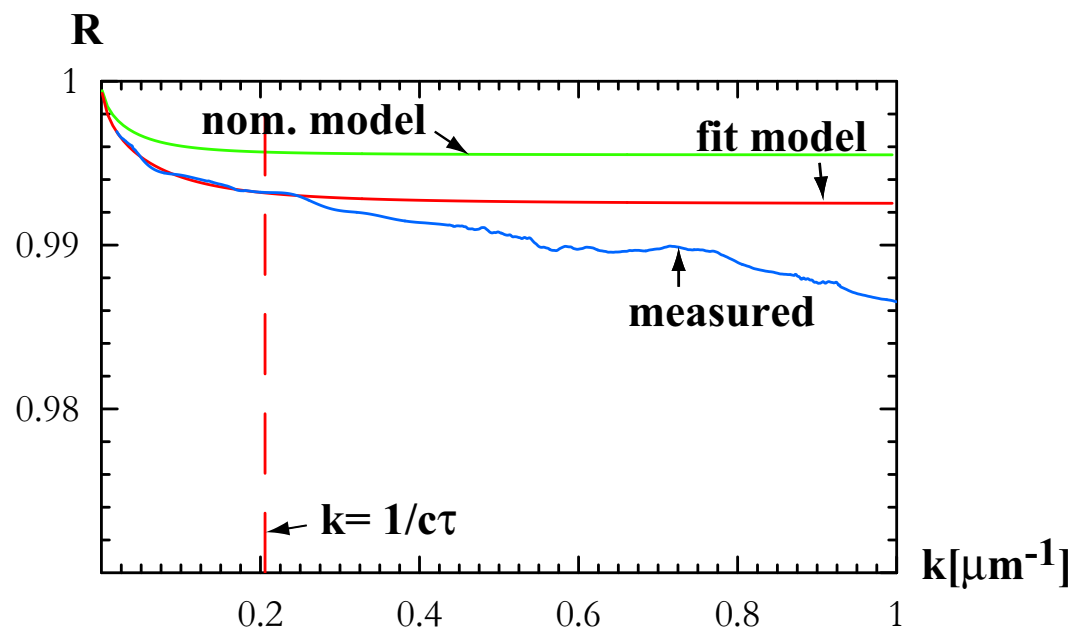

FIG. 4: Copper reflectivity: comparison of measurements (blue) with calculations using nominal $\sigma, \tau$ (green); and fitted values: 0.66 nominal $\sigma, 0.67$ nominal $\tau$ (red). The position of $k=1 / c \tau$ for the fit is also shown.

\section{CONCLUSION AND DISCUSSION}

In this note we analyzed reflectivity data, obtained recently at Brookhaven, for copper and evaporated aluminum samples. Our goal was to see whether or not the free-electron model used in resistive wall wakefield calculations for the LCLS undulator region [4] are valid. Thus, instead of as is typically done - taking the reflectivity measured over a large frequency range, together with the Kramers-Kroning integrals, to obtain electrical properties of a metal - we attempted here to fit the free-electron model directly over the frequency range of interest (the low frequency end of the data). The fitted dc conductivities and relaxation times when normalized to their nominal values, $\left(\sigma / \sigma_{\text {nom }}, \tau / \tau_{\text {nom }}\right)$, are approximately $(0.65$, $0.80)$ for $\mathrm{Al}$, and $(0.65,0.65)$ for $\mathrm{Cu}$. The fit over the low frequency range of the data is reasonably good; however, the fits do not cover the entire desired frequency $(k)$ range, $[0.06$, 0.6] $\mu \mathrm{m}^{-1}$ (only $40 \%$ of the range for $\mathrm{Cu}$ ).

At higher frequencies the measured $R$ varies linearly with frequency, unlike the constant dependence expected by the free-electron model. This point should be understood before giving too much credence to our results.

How do these results affect our confidence in our LCLS undulator wakefield calculations? 
For the undulator wakefield effect we are more sensitive to $\tau$, with low $\tau$ being better, than to $\sigma$. Aluminum was considered better than copper due to its low $\tau$ (and still reasonable $\sigma$ ), and the Brookhaven measurements give us some confidence that the aluminum wake calculations have validity. The measurements for copper, on the other hand, are in reasonable agreement with our assumed free-electron model only over the lower half of the desired frequency range; over the upper half the free-electron model does not seem to apply. The deviation in $R$ is small and may not have much effect. However, given the copper data, it is difficult to know precisely the implication for the wakefield of a copper pipe: reflectivity at normal incidence alone, without a model, is not enough information to make such a calculation.

\section{ACKNOWLEDGEMENTS}

We thank J. Welch for helpful discussions on the topic of this report.

\section{REFERENCES}

[1] J. Tu, Preliminary reflectivity data on metals, talk given during LCLS Week, January 25-27, 2005 at SLAC.

[2] E. Shiles et al., Physical Review B 22(4), 1612 (1980).

[3] N. Ashcroft and N. Mermin, Solid State Physics (Harcourt Brace College Publishers, Orlando, FL, 1976).

[4] K. L. Bane and G. Stupakov, Resistive wall wakefield in the LCLS undulator beam pipe, SLAC-PUB 10707, SLAC (2004).

[5] M. Ordal et al., Applied Optics 22(7), 1099 (1983).

[6] A. P. Lenham and D. M. Treherne, Journal Opt. Soc. Am. 55(5), 683 (1966). 